\title{
FREE NORMAL COMPLEMENTS AND THE UNIT GROUP OF INTEGRAL GROUP RINGS
}

\author{
ERIC JESPERS
}

\author{
(Communicated by Ronald M. Solomon)
}

\begin{abstract}
It is shown that there are only four finite groups $G$ with the property that $G$ has a nonabelian free normal complement in the unit group of the integral group $\mathbf{Z} \boldsymbol{G}$.
\end{abstract}

For finite commutative groups it is well known that the trivial units have a free abelian (normal) complement in the unit group of their integral group ring. Hence, it is natural to state the following problem: characterize the finite groups $G$ which have a nonabelian free normal complement in the unit group of the integral group ring $\mathbf{Z} G$. This question was posed to the author by $\mathbf{M}$. $\mathbf{M}$. Parmenter.

In this paper it is shown that the only groups with this property are:

1. $S_{3}=\left\langle a, b \mid a^{3}=1, b^{2}=1, b a=a^{-1} b\right\rangle$, the symmetric group of degree 3;

2. $D_{4}=\left\langle a, b \mid a^{4}=1, b^{2}=1, b a=a^{-1} b\right\rangle$, the dihedral group of order 8

3. $T=\left\langle a, b \mid a^{6}=1, b^{2}=a^{3}, b a=a^{5} b\right\rangle$, the dicyclic group of order 12 ; and

4. $P=\left\langle a, b \mid a^{4}=1, b^{4}=1, a b a^{-1} b^{-1}=a^{2}\right\rangle$, a group of order 16 .

Note that in $[4,6,9]$ it has been shown these groups indeed have the property under review.

Throughout $G$ denotes a finite group. In the first two lemmas we will make use of the following observation. Suppose $N$ is a nonabelian free normal complement of the trivial units in $\mathscr{U}(\mathbf{Z} G)$. Then, since $\mathscr{U}(\mathbf{Z} G) / N \cong \pm G$, in particular the factor group is finite, and because $N$ is nonabelian free, it follows that $\mathscr{U}(\mathbf{Z} G)$ does not contain a free abelian subgroup of rank two.

Lemma 1. If $\pm G$ has a nonabelian free normal complement in $\mathscr{U}(\mathbf{Z G )}$, then the order of an element in $G$ is either $1,2,3,4,5,6,8$, or 12 .

Proof. Let $g \in G$ have order $n$, and let $\langle g\rangle$ denote the cyclic subgroup generated by $g$. Since $\mathscr{U}(\mathbf{Z}\langle g\rangle)$ does not contain a free abelian subgroup of rank 2, it follows from Higman's result (cf. [15, Theorem II.3.1]) that the rank $r$ of

Received by the editors December 10, 1992.

1991 Mathematics Subject Classification. Primary 16U60, 16 S34.

This work is supported in part by NSERC Grant OGP0036631, Canada. 
the free abelian complement of the trivial units in $\mathscr{U}(\mathbf{Z}\langle g\rangle)$ is

$$
r=\frac{1}{2}\left(n+1+n_{2}-2 c\right)=0 \text { or } 1,
$$

where $c$ is the number of cyclic subgroups of $\langle g\rangle$ and $n_{2}$ is the number of elements of order 2 in $\langle g\rangle$.

The case $r=0$ is well known and yields $n=1,2,3,4$, or 6 . Assume now $r=1$, i.e., $n+n_{2}-2 c-1=0$. Now let $p$ be a prime number and $\alpha>1$ with $p^{\alpha} \mid n$. If $p \neq 2$ and $3 \neq p^{\alpha}$, then the above applied to the element $g^{n / p^{\alpha}}$ yields

$$
p^{\alpha}-2(\alpha+1)-1=0 .
$$

So $p^{\alpha}=2 \alpha+3$, and thus $p^{\alpha}=5$. It is easily verified that the identity $(*)$ fails for $n$ divisible by $24,16,15$, or 10 , i.e., in all cases the unit group contains a free abelian subgroup of rank at least 2 . Hence the only remaining possible values for $n$ are 5,8 , and 12 . The result follows.

Lemma 2. If $\pm G$ has a nonabelian free normal complement in $\mathscr{U}(\mathbf{Z} G)$, then $\mathbf{Q} G$ has a simple Wedderburn component which is isomorphic with $M_{2}(\mathbf{Q})$, a two-by-two matrix ring over the rationals, and all the other simple Wedderburn components are division rings.

Proof. Let $S$ be a simple component of $\mathbf{Q} G$. We first show that if $S \cong M_{n}(D)$, $D$ a division ring, and $n \geq 2$, then $n=2$. Indeed suppose $n \geq 3$, and let $E_{i, j}, 1 \leq i, j \leq n$, be a set of matrix units for $S$. So $E_{i, j}$ corresponds with a matrix which has 1 at the $(i, j)$-entry and zeros elsewhere. Since each $E_{i, j} \in \mathbf{Q} G$, there exists a positive integer $m$ such that $m E_{i, j} \in \mathbf{Z} G$. Clearly $\left(m E_{1,2}\right)^{2}=\left(m E_{1, n}\right)^{2}=0$. It follows easily that $1+m E_{1,2}$ and $1+m E_{1, n}$ generate a free abelian group of rank two, a contradiction. So $n=2$.

Next we show that $\mathbf{Q} G$ has at most one simple Wedderburn component which is not a division ring. Suppose there are two components $S_{1}$ and $S_{2}$ which are not division rings. As above, in each $S_{i} \cap \mathbf{Z} G$ there exist $0 \neq \alpha_{i}$ with $\alpha_{i}^{2}=0$. It again follows that $1+\alpha_{1}$ and $1+\alpha_{2}$ generate a free abelian group of rank 2, a contradiction.

Third we show that if a simple component $S \cong M_{2}(D), D$ a division ring, then $D=\mathbf{Q}$. If not, let $d \in(D \backslash \mathbf{Q})$. Then, with the above notation, let $m$ be a positive integer such that $m E_{1,2}, m d E_{1,2} \in \mathbf{Z} G$. As before, the units $1+m d E_{1,2}$ and $1+m E_{1,2}$ yield a contradiction.

So far we have shown that there is at most one component which is a matrix ring, and if such a component exists then it is isomorphic with $M_{2}(\mathbf{Q})$. It therefore remains to be shown that there exists a simple component which is a matrix ring. Assume the contrary, i.e., $\mathbf{Q} G$ is a direct sum of division rings. So $\mathbf{Q} G$ has no nonzero nilpotent elements. Theorem VI.1.17 in [15] therefore implies that $G$ is either abelian or Hamiltonian of order $2^{n} m, m$ odd such that the multiplicative order of 2 modulo $m$ is odd. The former case is excluded as then $\mathscr{U}(\mathbf{Z} G)$ is abelian, and no nonabelian free subgroup exists. In the latter case, it follows from the results of Moser [8] and Fein, Gordon, and Smith [2] (see also Proposition VI.1.13 and Theorem VI.1.15 in [15]) that if a prime $p$ divides $m$, then $p$ is congruent to 1 or 7 modulo 8 ; hence, $p \geq 7$. Lemma 1 yields $m=1$. Hence $G$ is a Hamiltonian 2-group, and thus by Higman's theorem, the units of $\mathbf{Z G}$ are trivial. This contradicts with the existence of a noncommutative free normal complement. 
The next two lemmas deal with proper subgroups of $G$.

Lemma 3. Suppose $\mathbf{Q G}$ has at most one simple component which is not a division ring, and suppose that if such a component exists then it is isomorphic with $M_{2}(\mathbf{Q})$. If $S$ is a proper subgroup of $G$, then $Q S$ is a sum of division rings and $S$ is either abelian or a Hamiltonian group. If, moreover, $S$ is nonabelian and every element of $S$ has order $1,2,3,4,5,6,8$, or 12 , then $S$ is a Hamiltonian 2-group.

Proof. In the last part of the proof of Lemma 2 it is shown that if $S$ is nonabelian and $\mathrm{Q} S$ is a sum of division rings then $S$ is Hamiltonian. Furthermore, $S$ is a Hamiltonian 2-group if every element has order $1,2,3,4,5,6,8$, or 12.

So the lemma will follow if we show that it is impossible to write $\mathbf{Q S}=$ $C \oplus B$, where $B$ is a sum of simple rings, $C=\mathrm{Q} S f \cong M_{n}(D), n>1$, $D$ a division ring, and $f$ a primitive central idempotent of $\mathbf{Q} S$. Suppose by contradiction that such a decomposition exists. For any primitive central idempotent $e$ of $\mathbf{Q} G$ either $e f=0$ or $e f \neq 0$. Assume $e f \neq 0$; then the natural ring homomorphism

$$
C \rightarrow \mathbf{Q G e}: \alpha \mapsto \alpha e
$$

is injective (as $C$ is simple). So $\mathbf{Q} G e$ contains nilpotent elements, and thus, by the assumption, $\mathrm{Q} G e \cong M_{2}(\mathbf{Q})$. It follows that

$$
\mathbf{Q} G e=C e=\mathbf{Q S f e} \text {. }
$$

So, $f e$ is the right identity of $\mathbf{Q} G e$. Hence $e=f e$. We have shown that if ef $\neq 0$, then $e f=e$ and $\mathbf{Q} G e=C e \cong M_{2}(\mathbf{Q})$. As $\mathbf{Q} G e$ has exactly one simple component which is isomorphic with $M_{2}(\mathbf{Q})$ there is only one $e$ with ef $\neq 0$. Since $f=\sum e f$, where the sum runs over all the primitive central idempotents of $\mathbf{Q} G$, we obtain $f=e f=e$. Consequently $\mathbf{Q} S f=\mathbf{Q} G f$, and thus $G f \subseteq \mathbf{Q} S$. However, this is impossible as $S$ is a proper subgroup of $G$.

Lemma 4. Suppose $\mathbf{Q} G$ has at most one simple component which is not a division ring, and suppose that if such a component exists then it is isomorphic with $M_{2}(\mathbf{Q})$. If $M$ is a maximal proper normal subgroup of $G$, then $G / M$ is $a$ cyclic group of prime order.

Proof. The simple components of $\mathbf{Q}(G / M)$ are simple components of $\mathbf{Q} G$. Hence $\mathbf{Q}(G / M)$ has at most one simple component which is not a division ring, and if so this component is isomorphic with $M_{2}(\mathbf{Q})$.

It is sufficient to show that the simple group $S=G / M$ is commutative. Suppose not; then $S$ contains an element of order 2 , say $s$. Since $S^{\prime}=S$, Lemma 1.2 in [1] yields

$$
\mathbf{Q} S \cong \mathbf{Q}(S / S) \oplus \omega(S) \cong \mathbf{Q} \oplus \omega(S),
$$

where $\omega(S)$ is the augmentation ideal and a sum of noncommutative simple rings. Since $S$ is embedded in each simple component $\mathrm{QS} e$ of $\omega(S)$, $e$ a primitive central idempotent, it follows that $e(1+s) \neq 0, e(1-s) \neq 0$, and $e(1+s)(1-s)=0$. So every simple component of $\omega(S)$ has nontrivial zero divisors and therefore is not a division ring. Hence there can be only one 
such simple component. So $\omega(S) \cong M_{2}(\mathbf{Q})$. Counting dimensions we obtain $|S|=1+\operatorname{dim}(\omega(S))=5$. So $S \cong \mathrm{Z}_{5}$, a contradiction.

We now show that elements of order 5 also are excluded in case the trivial units have a nonabelian free normal complement in the unit group. Furthermore, we exclude some division rings. By $\xi_{8}$ we denote a primitive root of unity of order 8 .

Lemma 5. If $\pm G$ has a nonabelian free normal complement in $\mathscr{U}(\mathbf{Z} G)$, then $G$ has no elements of order 5. Furthermore, if a division ring $D$ is a simple component of $\mathbf{Q} G$, then $D$ is neither $\mathbf{Q}\left(\xi_{8}\right)$ nor a quaternion algebra over $\mathbf{Q}(\sqrt{2})$.

Proof. Because of Lemma 2, $\mathbf{Q} G$ has exactly one simple component which is not a division ring, and this simple component is isomorphic with $M_{2}(\mathbf{Q})$.

Let $M$ be a maximal proper normal subgroup of $G$. By Lemma $4, G=$ $\langle M, g\rangle, g^{p} \in M, p$ a prime number.

We first show that 5 does not divide the order of $M$. Suppose this is false, then clearly $M$ is not a Hamiltonian 2-group. So, by Lemma 3, $M$ is abelian. Moreover, Lemma $1, M \cong C_{5} \times \cdots \times C_{5}$, a direct product of copies of the cyclic group $C_{5}$ of order 5 . Hence,

$$
\mathbf{Q} M \cong \mathbf{Q} \oplus \omega(M),
$$

and $\omega(M)$ is a direct sum of field extensions of degree 4 over $\mathbf{Q}$ (see, for example, [15, Proposition II.2.6]). The rational component corresponds with the primitive central idempotent $f=\frac{1}{|M|} \sum_{m \in M} m$. Note that $f$ is also central in $\mathbf{Q} G$ as $M$ is a normal subgroup. Let $e$ be the primitive central idempotent of $\mathbf{Q} G$ such that $\mathbf{Q} G e \cong M_{2}(\mathbf{Q})$. As $\mathbf{Q} G e$ is noncommutative of dimension 4 over the rationals, it does not contain a field extension of degree 4 over $\mathbf{Q}$. Hence $\omega(M) e=0$. So

$$
\mathbf{Q} G e=\sum_{1 \leq i \leq p}\left(\mathbf{Q} f g^{i} e\right)
$$

It follows that $\mathbf{Q} G e$ is commutative, a contradiction. Hence we have shown that 5 does not divide $|M|$.

Second, we show that 5 does not divide $|G / M|$. Since $G^{\prime} \subseteq M$, it follows from the first part that it is sufficient to show that 5 does not divide $\left|G / G^{\prime}\right|$. Suppose the contrary. Again

$$
\mathbf{Q} G \cong \mathbf{Q}\left(G / G^{\prime}\right) \oplus \omega_{G}\left(G^{\prime}\right),
$$

where $\omega_{G}\left(G^{\prime}\right)$ is the augmentation ideal determined by the normal subgroup $G^{\prime}$. As $\mathbf{Q}\left(G / G^{\prime}\right)$ is abelian, it follows that $\omega_{G}\left(G^{\prime}\right)$ has one simple component isomorphic with $M_{2}(\mathbf{Q})$. Hence, as before, there exists a nonperiodic unit element in $\mathbf{Z} G$ that is of the form $1+\alpha$, where $\alpha \in \mathbf{Q} G e$, and $\alpha^{2}=0$. Also, as $\left|G / G^{\prime}\right|$ is divisible by 5 , it follows from Higman's results (cf. [15, Theorem II.3.1]) that $\mathscr{U}\left(\mathbf{Z}\left(G / G^{\prime}\right)\right)$ contains a unit $\bar{u}$ of infinite order. Let $u$ be a preimage of $\bar{u}$ in $\mathbf{Z} G$, and let $\widehat{G^{\prime}}=\frac{1}{\left|\widehat{G}^{\prime}\right|} \sum_{g \in G^{\prime}} g$. Then $\omega_{G}\left(G^{\prime}\right)=\mathbf{Q} G\left(1-\widehat{G^{\prime}}\right)$ and

$$
v=u \widehat{G^{\prime}} \oplus\left(1-\widehat{G^{\prime}}\right)
$$

is a unit in $\mathbf{Z} G \widehat{G^{\prime}} \oplus \mathbf{Z} G\left(1-\widehat{G^{\prime}}\right)$. Since $\mathbf{Z} G \subseteq \mathbf{Z} G \widehat{G^{\prime}} \oplus \mathbf{Z} G\left(1-\widehat{G^{\prime}}\right)$ are $\mathbf{Z}$-orders, it follows from [15, (2.9), p. 49] that $v^{k}$, for some $k \geq 1$, is a nonperiodic unit 
in $\mathbf{Z G}$. It is easily verified that $v^{k}$ and $1+\alpha$ generate a free abelian group of rank two, a contradiction.

Finally, assume that $e$ is a primitive central idempotent such that $\mathbf{Q} G e=D$ is either a quaternion algebra over $\mathbf{Q}(\sqrt{2})$ or $\mathbf{Q}\left(\xi_{8}\right)$. Then, $Z(D)$ contains a Z-order (either $\mathbf{Z}[\sqrt{2}]$ or $\mathbf{Z}\left[\xi_{8}\right]$ ) which contains an element $u$ of infinite order. As in the previous argument, for some $k>0,(1-e)+u^{k}$ is a unit of infinite order in $\mathbf{Z G}$; again yielding a contradiction.

Next we will show that, under the previously considered assumptions, $G$ has a proper normal subgroup of index 2 in $G$. But first we need the following lemma, which is a consequence of [3, Theorem 2, p. 59]. A proof (in a more general situation) can be found in [5].

Lemma 6. Let $e$ be a primitive central idempotent of $\mathbf{Q} G$ such that $\mathbf{Q} G e \cong$ $M_{2}(\mathbf{Q})$. If $f$ is a noncentral idempotent in $\mathbf{Q} G e$, then there exist matrix units $E_{u, v}, 1 \leq u, v \leq 2$, such that $f=E_{1,1}$, and $\mathbf{Q} G e$ can be considered as $a$ matrix ring over $\mathbf{Q}$ which is the centralizer of all $E_{u, v}$.

Lemma 7. Suppose $\mathbf{Q} G$ has exactly one simple component which is not a division ring, and suppose this simple component is isomorphic with $M_{2}(\mathbf{Q})$. If $x \in G$ and $M$ is a maximal proper normal subgroup of $G$ with $x^{3}=1$ and $G=$ $\langle M, x\rangle$, then the cyclic group $\langle x\rangle$ is normal in $G$.

Proof. It is sufficient to show that the idempotent $f=\frac{1}{3}\left(1+x+x^{2}\right)$ is central. To do so we show that for every central primitive idempotent $e \in \mathbf{Q} G$, either $e f=0$ or $e f=f$. As $e f$ is an idempotent the latter is clear when $\mathbf{Q} G e$ is a division ring. So we are left with the case that $\mathbf{Q} G e \cong M_{2}(\mathbf{Q})$. If $e f$ is central in the simple algebra $\mathbf{Q} G e$ then obviously $e f=0$ or $e f=e$. So suppose that ef is not central. By Lemma 6, $\mathbf{Q} G e$ contains matrix units $E_{1,1}, E_{1,2}, E_{2,1}$, and $E_{2,2}$ with $E_{1,1}=e f$ and $E_{2,2}=e-e f$. As $x=f x f+(1-f) x(1-f)$, we obtain

$$
e x=e f x f e+(e-e f) x(e-e f) .
$$

So ex corresponds with a matrix of the type $\left[\begin{array}{ll}s & 0 \\ 0 & t\end{array}\right], s, t \in \mathbf{Q}$. Since $x^{3}=1$, it follows that $s^{3}=t^{3}=1$. Therefore, $s=t=1$. Hence $e x=e$, and $M_{2} \cong \mathbf{Q} G e$ is a homomorphic image of $\mathbf{Q} M$. However, this is impossible because of Lemma 3; the result follows.

Lemma 8. Suppose $\mathbf{Q} G$ has exactly one simple component which is not a division ring, and suppose this simple component is isomorphic with $M_{2}(\mathbf{Q})$. If every element of $G$ has order $1,2,3,4,6,8$, or 12 , then $G$ has a maximal proper normal subgroup $M$ such that $G / M$ is a cyclic group of order 2 .

Proof. First of all notice that $G$ is a group of even order, as $\mathbf{Q} G$ contains $M_{2}(\mathbf{Q})$ as a simple component.

Because of the assumptions and Lemma 4, $G$ has a maximal proper normal subgroup $M$ such that $G / M \cong C_{2}$ or $G / M \cong C_{3}$. In the former case the result follows. So assume that $G=\langle M, x\rangle$, with $x^{3}=1, x^{6}=1$, or $x^{12}=1$. Replacing, if necessary, $x$ by $x^{2}$ or $x^{4}$ we may assume that $x^{3}=1$. By Lemma 7, $\langle x\rangle$ is a normal subgroup of $G$. As $\mathbf{Q}(G /\langle x\rangle)$ is a homomorphic image of $\mathbf{Q} G$, one obtains that $\mathbf{Q}(G /\langle x\rangle)$ has at most one simple component which is not a division ring, and if such a component exists then it is isomorphic with 
$M_{\mathbf{2}}(\mathbf{Q})$. If $\mathbf{Q}(G /\langle x\rangle)$ has no matrix-component, then, as in the first part of the proof of Lemma 3, $G /\langle x\rangle$ is Abelian or a Hamiltonian 2-group. Consequently there exist a maximal proper normal subgroup $N$ of $G$ containing $\langle x\rangle$ such that $G / N \cong C_{2}$. On the other hand, if $\mathbf{Q}(G /\langle x\rangle)$ contains $M_{2}(\mathbf{Q})$ as a simple component, then $|G /\langle x\rangle|<|G|$ and the result follows by induction on the order of the group.

Now we show that $G$ is generated by two elements in case $\mathbf{Q} G$ has the appropriate Wedderburn decomposition.

Lemma 9. Suppose $\mathbf{Q} G$ contains a primitive central idempotent $e$ such that $\mathbf{Q G e} \cong M_{2}(\mathbf{Q})$. Further assume that every other simple component of $\mathbf{Q} G$ is $a$ division ring. If $a, b \in G$ are such that ae and be do not commute, then $G=\langle a, b\rangle$.

Proof. Let $F$ be the Q-subalgebra of $\mathrm{Q} G e$ generated by $b e$, and let $H$ be the cyclic subgroup of $G e$ generated by $b e$. Then $F$ is a homomorphic image of the commutative semisimple Artinian group algebra $\mathbf{Q} H$. Hence $F=F_{1} \oplus$ $\cdots \oplus F_{k}$, a direct sum of fields. Let $f_{i}$ denote the identity of $F_{i}$. Note that $e=f_{1}+\oplus \cdots \oplus f_{k}$. We consider two cases.

Case 1: $k \geq 2$. Then $f_{1} \neq 0, f_{1} \neq e$, and thus $f_{1}$ is not central in $\mathbf{Q} G e$. Hence by Lemma 6, QGe has a set of matrix units $E_{1,1}, E_{1,2}, E_{2,1}, E_{2,2}$ with $f_{1}=E_{1,1}$ and $f_{2}=E_{2,2}$ (note that $k=2$ follows and also $F_{1} \cong \mathbf{Q} \cong F_{2}$ ). With respect to these matrix units the elements of $F$ are represented by matrices of the form $\left[\begin{array}{ll}x & 0 \\ 0 & y\end{array}\right], x, y \in \mathbf{Q}$. Further let $a e$ be represented by the matrix $\left[\begin{array}{c}u v \\ s\end{array}\right]$, where $u=f_{1} a f_{1} e, v=f_{1} a f_{2} e, s=f_{2} a f_{1} e$, and $t=f_{2} a f_{2} e$. Now, $v \neq$ 0 or $s \neq 0$ because otherwise $a e$ and $b e$ commute. Hence the Q-subalgebra of $\mathbf{Q} G e$ generated by $a e$ and be contains nilpotent elements. As $\mathbf{Q} G e$ is 4dimensional, it follows that this subalgebra equals $\mathbf{Q} G e$. Consequently, $\mathbf{Q} G e$ is an epimorphic image of the group algebra $\mathbf{Q}\langle a, b\rangle$. Lemma 3 yields that $G=\langle a, b\rangle$.

Case 2: $k=1$. So $F$ is a field. Note that $F$ is at least 2-dimensional as a Q-vector space, because $b e$ is not central and therefore does not belong to $\mathbf{Q} e$. Also, as $a e$ and $b e$ do not commute, $a e \notin F$. So $\operatorname{dim}_{\mathbf{Q}}(\mathbf{Q}\langle a, b\rangle e) \geq 4$. Hence $\mathbf{Q}\langle a, b\rangle \boldsymbol{e}=\mathbf{Q} G e \cong M_{2}(\mathbf{Q})$. Again, by Lemma 3, it follows that $\langle a, b\rangle=G$.

Theorem 10. Let $G$ be a finite group. The following conditions are equivalent:

(1) $\pm G$ has a nonabelian free normal complement in $\mathscr{U}(\mathbf{Z} G)$.

(2) Every element of $G$ has order $1,2,3,4,6,8$, or 12 and $\mathbf{Q} G \cong$ $M_{2}(\mathbf{Q}) \oplus\left(\bigoplus_{i} D_{i}\right)$, where each $D_{i}$ is a division ring and $D_{i}$ is neither $\mathbf{Q}\left(\xi_{8}\right)$ nor a quaternion algebra over $\mathbf{Q}(\sqrt{2})$.

(3) $G$ is isomorphic with either $S_{3}, D_{4}, T$, or $P$.

Proof. That (1) implies (2) follows from Lemmas 1, 2, and 5. In the introduction it is mentioned that (3) implies (1) is known. So we now prove (2) implies (3). By Lemma 8, let $M$ be a maximal proper normal subgroup of $G$ with $G / M \cong C_{2}$. Write $G=\langle M, x\rangle, x^{2} \in M$. By Lemma $3, M$ is either abelian or a Hamiltonian 2-group.

We first deal with the case that $M$ is abelian. Suppose $M /(Z(G) \cap M)$ is generated by the cosets $m_{i}(Z(G) \cap M), 1 \leq i \leq k$. Let $e$ be the primitive central idempotent of $\mathbf{Q} G$ such that $\mathbf{Q} G e \cong M_{2}(\mathbf{Q})$. Then, for some $i$, say 
$i=1, m_{i} e$ and $x e$ do not commute. Lemma 9 yields that $G=\left\langle m_{1}, x\right\rangle$. We simply denote $m=m_{1}$. Since $M$ is abelian and $x^{2} \in M, m\left(x m x^{-1}\right)=$ $z \in(Z(G) \cap M)$ or, equivalent, $m^{-1} x m=z m^{-2} x$. If $m^{2} \in Z(G)$, then $G / Z(G) \cong C_{2} \times C_{2}$. It follows easily from the assumptions on the order of the elements and the results in [4] that $G \cong D_{4}$ or $G \cong P$. So for the remainder of this case we assume that $m^{2} \notin Z(G)$. Let $S=\left\langle m^{2}, x\right\rangle$. Note that $S$ is a nonabelian subgroup of $G$. Again we deal with two cases: (i) $S \neq G$ and (ii) $S=G$.

(i) $S \neq G$. Hence by Lemma $3, S=Q_{8} \times C_{2} \times \cdots \times C_{2}$, where $Q_{8}$ is the quaternion group of order 8 . Clearly $\left|S^{\prime}\right|=2$ and $S / S^{\prime} \cong C_{2} \times \cdots \times C_{2}$. Since $S$ is generated by two elements only, $\left|S / S^{\prime}\right|=2$ or 4 , and thus, because $S$ is not abelian, $|S|=8$. If $S$ is normal in $G$ (i.e., $z \in S$ ), then $G / S \cong C_{2}$ and thus $|G|=16$. From the assumptions and the study of the groups of order 16 in [7] we obtain that $G \cong P$. On the other hand, if $S$ is not normal in $G$, then $S \subset T=\left\langle m^{2}, x, z\right\rangle \subset G$. Hence, as in the previous argument, $S$ and $T$ are Hamiltonian 2-groups, $|S|=8,|T|=16$, and thus $|G|=32$. So $S^{\prime}=T^{\prime}$ is normal in $G$. It is then shown, as in the last part of the proof for $M$ a Hamiltonian 2-group, that this case is impossible.

(ii) $S=G$. So $m \in\left\langle m^{2}, x\right\rangle$. Consequently $m(Z(G) \cap M)$ has odd order in $M /(Z(G) \cap M)$. The assumptions therefore yield that $m^{3}$ is central and $m$ has order 3,6 , or 12 . It follows that $z^{6}=1$. Hence

$$
x(m z)^{4}=(m z)^{-4} x .
$$

Replacing $m^{2}$ by $(m z)^{4}$ (this is allowed by Lemma 9) we may assume $G=$ $\left\langle m^{2}, x\right\rangle$ with $x m^{2}=m^{-2} x, m^{2}$ of order 3 . So $\left\langle m^{2}\right\rangle$ is a normal subgroup of $G$. The order of $x$ is either $2,3,4,6,8$, or 12 . It cannot be 3 , because otherwise $G$ is a group of order 9 , and thus commutative. Also the order is not 6 , for else $S_{3} \cong\left\langle x^{3}, m^{2}\right\rangle$ is a proper subgroup of $G$, in contradiction with Lemma 3. Also order 8 is impossible. For if not, then $\mathbf{Q}\left(G /\left\langle m^{2}\right\rangle\right) \cong$ $\mathbf{Q}\left(\left\langle x \mid x^{8}=1\right\rangle\right)$ (and thus also $\left.\mathbf{Q} G\right)$ has a simple component of the type $\mathbf{Q}\left(\xi_{8}\right)$, a contradiction. Further, order 12 is also impossible, for otherwise $\left\langle x^{3}, m^{2}\right\rangle$ is a noncommutative proper subgroup of order 12; this is impossible because of Lemma 3. On the other hand, if the order is 2 , then $|G|=6$, and $G \cong S_{3}$. If the order is 4 , then $|G|=12$. The only noncommutative groups of order 12 are $S_{3} \times C_{2}, A_{4}$ the alternating group of degree 4 , and $T$. It is well known that the two former cases do not have the required Wedderburn decomposition. So $G \cong T$.

This finishes the proof for $M$ abelian.

Suppose now that $m$ is a Hamiltonian 2-group. Then $M^{\prime}$ is a normal subgroup of $G$ and $\left|M^{\prime}\right|=2$. Further

$$
\mathbf{Q} G \cong \mathbf{Q}\left(G / M^{\prime}\right) \oplus \omega_{M^{\prime}}(M) \mathbf{Q} G .
$$

As $\omega_{M}\left(M^{\prime}\right)$ is a direct sum of quaternion algebras over the rationals, it follows that every simple component of $\omega_{M^{\prime}}(M) \mathbf{Q} G$ is not isomorphic with $M_{2}(\mathbf{Q})$. Hence $G / M^{\prime}$ satisfies the same assumptions as $G$. Since $M / M^{\prime}$ is abelian and $G$ is a 2-group, it follows from the previous case that $\left|G / M^{\prime}\right|=8$ or 16 . So $|G|=16$ or $|G|=32$. The groups of order 16 have been studied in [7], and it follows that $G \cong P$ if $|G|=16$. We now finish the proof by showing that $|G|=32$ is impossible. Suppose not; then $G / M^{\prime} \cong P=\langle a, b| a^{4}=1, b^{4}=1$, 
$\left.a b a^{-1} b^{-1}=a^{2}\right\rangle$. The elements of order 2 in $P$ are $a^{2}, b^{2}, a^{2} b^{2}$. However, $M / M^{\prime} \cong C_{2} \times C_{2} \times C_{2}$ has 7 elements of order 2, a contradiction.

During the past few years there has been a lot of progress on determining finitely many generators of a subgroup of finite index of the unit group (see, for example, $[5,10,12,13,14])$. In particular, subgroups of finite index are a bit better understood. Hence, one might ask for a characterization of those finite groups $G$ which are such that $\mathscr{U}(\mathbf{Z G})$ has a nonabelian free subgroup of finite index. Obviously, if $G$ is such a group then the unit group still does not contain a free abelian subgroup of rank 2. Therefore, our proofs remain valid in this situation. So $S_{3}, D_{4}, T$, and $P$ are the only groups having this property.

\section{REFERENCES}

1. D. B. Coleman, Finite groups with isomorphic group algebras, Trans. Amer. Math. Soc. 105 (1962), 1-8.

2. B. Fein, B. Gordon, and J. H. Smith, On the representation of -1 as a sum of two squares in an algebraic number field, J. Number Theory 3 (1971), 310-315.

3. N. Jacobson, Structure of rings, Amer. Math. Soc. Colloq. Publ., vol. 37, Amer. Math. Soc., Providence, RI, 1964.

4. E. Jespers and G. Leal, Describing units of integral group rings of some 2-groups, Comm. Algebra 19 (1991), 1809-1827.

5. $\_$, Generators of large subgroups of the unit group of integral group rings, Manuscripta Math. 78 (1993), 303-315.

6. E. Jespers and M. M. Parmenter, Bicyclic units in $\mathrm{ZS}_{3}$, Bull. Soc. Math. Belg. Sér. B 44 (1992), 141-145.

7. _ Units of group rings of groups of order 16, Glasgow Math. J. 35 (1993), 367-379.

8. C. Moser, Representation de -1 comme somme de carres dans un corps cyclotomique quelconque, J. Number Theory 5 (1973), 139-141.

9. M. M. Parmenter, Free torsion-free normal complements in integral group rings, Comm. Algebra (to appear).

10. J. Ritter and S. K. Sehgal, Certain normal subgroups of units in group rings, J. Reine Angew. Math. 381 (1987), 214-220.

11. $\ldots$, Construction of units in integral group rings of fintie nilpotent groups, Trans. Amer. Math. Soc. 324 (1991), 603-621.

12. _ Generators of subgroups of $U(\mathrm{Z} G)$, Contemp. Math., vol. 93, Amer. Math. Soc., Providence, RI, 1989, pp. 331-347.

13. Construction of units in group rings of monomial and symmetric groups, J. Algebra 142 (1991), 511-526.

14. _ Units of group rings of solvable and Frobenius groups over large rings of cyclotomic integers, J. Algebra (to appear).

15. S. K. Sehgal, Topics in group rings, Marcel Dekker, New York, 1978.

Department of Mathematics and Statistics, Memorial University of Newfoundland,

St. John's, Newfoundland, Canada AIC 5S7

E-mail address: EJESPERSQMORGAN.UCS.MUN.CA 